\title{
Activation of pluripotency-associated genes in mouse embryonic fibroblasts by non-viral transfection with in vitro-derived mRNAs encoding Oct4, Sox2, Klf4 and cMyc
}

\author{
Geertrui Tavernier ${ }^{a}$, Katharina Wolfrum ${ }^{b}$, Joseph Demeester ${ }^{a}$, Stefaan C. De Smedt ${ }^{a, *}$, James Adjaye ${ }^{b}$, \\ Joanna Rejman ${ }^{\mathrm{a}, *}$ \\ ${ }^{a}$ Research Group on Nanomedicines, Ghent University, Harelbekestraat 72, Ghent, Belgium \\ ${ }^{\mathrm{b}}$ Molecular Embryology and Aging Group, Department of Vertebrate Genomics, Max Planck Institute for Molecular Genetics, Ihnestrasse 63, Berlin, Germany
}

\section{A R T I C L E I N F O}

\section{Article history:}

Received 11 August 2011

Accepted 23 September 2011

Available online 10 October 2011

\section{Keywords:}

mRNA

Reprogramming

Non-viral

Liposome

Oct4

\begin{abstract}
A B S T R A C T
The first successful reprogramming of differentiated cells to a pluripotent state was done by retroviral introduction of four transcription factors (Oct4, Sox2, Klf4, cMyc) by the group of Yamanaka in 2006. Since then, scientists all over the world have attempted various methods to avoid insertional mutagenesis, a major limitation of the retrovirus-based method, however no technique was found to completely avoid DNA integration. Recently, a non-viral mRNA-based approach, inherent to avoid genomic integration, was implemented to generate stem cell-like cells, yet, seventeen daily transfections were required, inducing substantial stress on the cells. In this work, we demonstrate successful activation of pluripotency-associated genes in mouse embryonic fibroblasts by means of cationic lipid-mediated introduction of mRNAs encoding the four factors. Moreover, our transfection protocol required maximally three transfections. Up-regulation of the transfected factors as well as Nanog and SSEA-1, typical mouse pluripotency markers, was detected already after the first transfection. Nuclear localization of the introduced factors was confirmed. Positive alkaline phosphatase staining of cell clusters further confirmed the onset of the reprogramming process. In conclusion, the transfection method presented here holds great promise for safe generation of induced pluripotent stem cells of mouse origin.
\end{abstract}

(c) 2011 Elsevier Ltd. All rights reserved.

\section{Introduction}

The reprogramming of somatic cells to generate so-called induced pluripotent stem cells (iPSCs) is a fairly recent and promising development in regenerative medicine. These cells are very similar to embryonic stem cells (ESCs) and have the potential to self-renew and differentiate into specific cell types of all three germ layers. The groundbreaking work of Yamanaka's group demonstrated the possibility of reprogramming by retrovirus-mediated introduction of four transcription factors (Oct4, Sox2, Klf4 and cMyc) in murine [1] and later also human [2,3] fibroblasts. It was later shown that iPSCs could be generated even without exogenous cMyc [4], only with Oct4 and Sox2 [5] and that cMyc and Klf4 could be replaced by Nanog and Lin28 when reprogramming human cells [3]. Although successful, the major disadvantage of this method is

\footnotetext{
* Corresponding authors.

E-mail addresses: stefaan.desmedt@ugent.be (S.C. De Smedt), j_rejman@ hotmail.com (J. Rejman).
}

the abundant insertional mutagenesis seen in the resulting iPSCs. These unpredictable DNA integrations can lead to malignancy and other undesired effects. Moreover, they can bias or even silence the differentiation potential of the resulting iPSCs [6]. Therefore, research groups all over the world have focused on generating iPSCs trying both to minimize genome integrations as well as to increase efficiency (only $0.01-0.1 \%$ of the cells become iPSCs with the retroviral approach) [6]. Genes encoding reprogramming factors have been introduced using non-integrating viral (episomal) vectors [7,8], by repeated non-viral transfections [9] or by means of excisable vectors which can remove most, but not all, transgene sequences once the cells have changed to a pluripotent state [10,11]. Despite the significant reduction in genomic integration sites accomplished by these new approaches, the danger of insertional mutagenesis could not be eliminated completely. Alternatively, transduction of recombinant transcription factor proteins was reported to produce iPSCs [12]. This method however, has been shown to have low efficiency and bacterial posttranslational modification of proteins revealed essential shortcomings. 
An mRNA-based approach was recently proposed as a solution to overcome all the above-mentioned challenges and drawbacks inherent to viral and episomal plasmid-based reprogramming protocols. Indeed, the use of mRNA avoids the risk of modifying the host genome and the method is simple and less time-consuming than previously explored approaches. Plews et al. [13] demonstrated the activation of pluripotency-associated genes in human fibroblasts by introducing in vitro transcribed mRNA encoding the reprogramming factors OCT4, SOX2, KLF4 and cMYC into the cells by non-viral means. Using the same technique, Warren and colleagues [14] were able to generate iPSCs with surprisingly high efficiency. Yet, transfections had to be performed daily over a period of seventeen days, which renders the method highly stress-inducing for the cells, having to deal with large amounts of the cationic carrier lipofectamine. Here, we demonstrate successful onset of the reprogramming process in mouse embryonic fibroblasts by means of cationic lipid-mediated introduction of mRNAs encoding the Yamanaka factors.

\section{Materials and methods}

\subsection{Cell culture}

Mouse embryonic fibroblasts (MEFs) in which Neomycin-resistance is driven by the Oct4 promoter and enhancer, were purchased at Stemgent (via Miltenyi, Bergisch Gladbach, Germany) and cultured in embryonic stem cell (ESC)-qualified DMEM (Millipore, Overijse, Belgium) supplemented with 10\% ES-qualified serum (Millipore), $1 \%$ non-essential amino acids (Millipore) $1 \%$ mercaptoethanol (Millipore), $2 \mathrm{mM}$ L-glutamine (Invitrogen-Gibco, Merelbeke, Belgium), and $100 \mu \mathrm{g} / \mathrm{ml}$ penicillin/streptomycin (Invitrogen-Gibco) (MEF medium) at $37^{\circ} \mathrm{C}, 5 \% \mathrm{CO}_{2} .10^{5} \mathrm{U} / \mathrm{ml}$ ES-qualified LIF (Leukaemia Inhibitory Factor, Millipore) was added to the medium at time points indicated in Fig. 3 (MEF + LIF medium). Also depicted in this figure are the time points at which treated cells were plated on feeder MEFs (mitomycin C treated, purchased from Millipore).

\section{2. mRNA synthesis}

Appropriate plasmids (containing a T7 RNA polymerase promoter) encoding either a single reprogramming factor (Oct4, Sox2, cMyc or Klf4) or containing all factors separated by the viral $2 \mathrm{~A}$ peptide were purchased from Addgene (Cambridge, USA). The plasmid encoding green fluorescent protein (GFP) (pGEM4Z-EGFP-A64, kindly provided by Prof. Dr. E. Gilboa, Duke University Medical Center, Durham, NC, USA) was previously described by Nair et al. [15]. To produce mRNAs, plasmids were first purified using a QIAquick PCR purification kit (Qiagen, Venlo, Netherlands) and linearized using restriction enzymes (HindIII for the plasmid encoding cMyc; SpeI for the plasmid encoding GFP; NotI - for all the other plasmids). Linearized plasmids were used as templates for the in vitro transcription reaction using the T7 mMessage mMachine kit according to the manufacturer's instructions (Ambion, Lennik, Belgium). This resulted in production of mGFP that was both capped and polyadenylated. All the other mRNAs are capped and subsequently polyadenylated with a poly(A) tailing kit provided by Ambion. mRNAs were purified by DNase I digestion followed by $\mathrm{LiCl}$ precipitation and $70 \%$ ethanol wash. The mRNA concentration was determined by measuring the absorbance at $260 \mathrm{~nm}$. mRNA was stored in small aliquots at $-80^{\circ} \mathrm{C}$ at a concentration of $1 \mu \mathrm{g} / \mu \mathrm{l}$.

\section{3. mRNA transfection}

MEFs (150,000 cells/well) were seeded one day prior to the experiment in 6-well plates. Either $4 \mu \mathrm{g}$ of mGFP or mOSKM (one mRNA-molecule encoding all four reprogramming factors separated by the viral peptide $2 \mathrm{~A}$ ) or a $4 \mu \mathrm{g}$ mixture of the four separate mRNAs (equal amounts of mOct4, mSox2, mKlf4, mcMyc), pre-diluted in $46 \mu \mathrm{l}$ OptiMem (Invitrogen-Gibco), were complexed with $4 \mu \mathrm{l}$ of Lipofectamine RNAiMAX (LF) $(1 \mathrm{mg} / \mathrm{ml}$, purchased from Invitrogen), also pre-diluted in $46 \mu \mathrm{l}$ OptiMem. After $10 \mathrm{~min}$ incubation at RT, $900 \mu \mathrm{l}$ of OptiMem were added and this solution was added to the cells (after removal of growth medium). After $2 \mathrm{~h}$ of incubation with the cells, the complexes were removed and regular growth medium was added. Up to three consecutive transfections were executed (schematically presented in Fig. 3).

\subsection{Analysis of GFP expression}

Images showing mRNA-induced GFP expression were taken with a confocal microscope LSM 510 (Zeiss, Oberkochen, Germany). To assess the number of GFPpositive cells, culture medium was removed from the wells and the cells were washed with PBS. After detaching the cells with trypsin $(0.05 \%$, Gibco/Invitrogen) and centrifugation, the cells were re-suspended in flow buffer (PBS containing $1 \%$ BSA and $0.1 \%$ azide). The samples were kept on ice until GFP expression was evaluated by a Beckman Coulter Flowcytomer FC500, equipped with a $488 \mathrm{~nm}$ laser. (Zeiss).

Images showing GFP expression were taken with a confocal microscope LSM 510

\subsection{Immunostaining and flow cytometry analysis}

To evaluate the expression of the factors quantitatively, culture medium was removed from the wells and the cells were washed with PBS. After detaching the cells with trypsin (0.05\%) (Invitrogen-Gibco) and centrifugation, the cells were resuspended in Perm/Wash buffer (BD, Bioscience Pharmingen, Belgium). After washing, 5\% goat serum (Invitrogen-Gibco) was added for $20 \mathrm{~min}$ at $4{ }^{\circ} \mathrm{C}$ to block unspecific binding. Cells were stained in Perm/Wash buffer with primary antibodies for $30 \mathrm{~min}$ at $4{ }^{\circ} \mathrm{C}$ and subsequently washed with Perm/Wash Buffer and stained with secondary antibody for $30 \mathrm{~min}$ at $4{ }^{\circ} \mathrm{C}$, protected from light. After final washing in Perm/Wash buffer, the cells were re-suspended in flow buffer (PBS containing 1\% BSA and $0.1 \%$ azide) and the samples were kept on ice until the expression was evaluated by analysis in a Beckman Coulter Flowcytometer FC500, equipped with a $488 \mathrm{~nm}$ laser. The expression levels are expressed as mean intensity per cell as compared to control samples. Antibodies used to stain the introduced factors were purchased from Abcam, primary antibodies included Oct4 (Cat. No. ab18976), Sox2 (Cat. No. ab59776), Klf4 (Cat. No. ab26648), cMyc (Cat. No. ab51154) and the secondary antibody was conjugated with Cy5 (Cat. No. ab6564). The primary antibodies for Nanog (Cat. No. 09-0020) and for SSEA-1 (Cat. No. 09-0005) and the secondary antibody conjugated with Dylight-488 (Cat. No. 09-0033) were purchased from Stemgent (via Miltenyi).
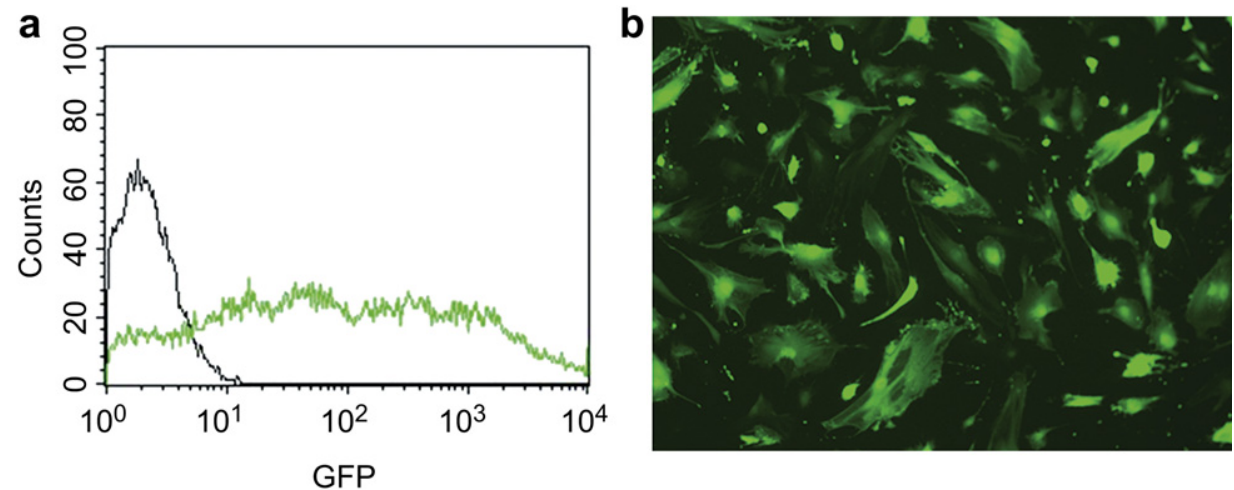

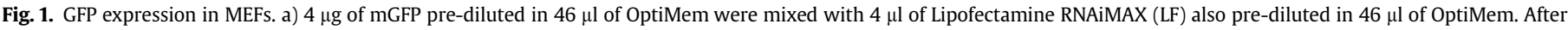

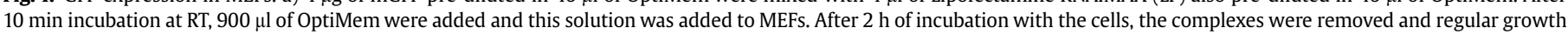

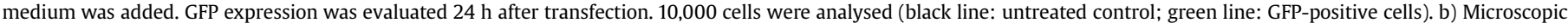

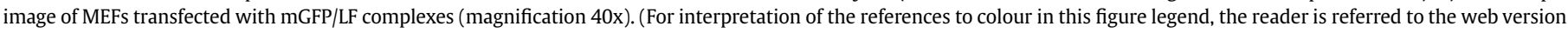
of this article.) 


\subsection{Immunostaining and microscopy analysis}

To ensure the nuclear expression of the reprogramming factors, by means of immunocytochemical analysis, cells were fixed with $4 \%$ paraformaldehyde (Science Services, Munich, Germany) for 20 min at RT, washed two times with phosphatebuffered saline (PBS) and blocked with 5\% goat serum (Invitrogen-Gibco) and 1\% Triton X-100 (Sigma Aldrich, Munich, Germany). Nuclei were counterstained with DAPI (200 ng/ml, Invitrogen, Carlsbad, CA, USA). Cells were visualized using a confocal microscope LSM 510 (Zeiss). The same primary antibodies as for immunostaining for flow cytometry (above) were used, the secondary antibody conjugated with Dylight-488 was purchased from Abcam (Cat. No. ab96923).

\subsection{Alkaline phosphatase (ALP) staining}

The ALP assay was performed using the Stemgent alkaline phosphatase staining kit, according to the manufacturer's instructions (via Miltenyi). The samples were analyzed with a Nikon C1 confocal laser scanning microscope (Nikon Benelux, Brussels, Belgium).

\section{Results}

3.1. Transfection of mouse embryonic fibroblasts with mRNA encoding green fluorescent protein or reprogramming factors

MEFs were transfected with mRNA encoding green fluorescent protein (mGFP) according to the lipofection protocol described in the MM section. Fig. 1 shows the flow cytometry and microscopy analysis of GFP-positive MEFs $24 \mathrm{~h}$ after transfection. At this time point, GFP was expressed abundantly in more than $86 \%$ of the cells. We next transfected MEFs, either with one mRNA-molecule encoding all four reprogramming factors separated by the viral peptide 2A (mOSKM) or with equal amounts of four different mRNAs each encoding one factor (mOct4, mSox2, mKlf4 and mcMyc). To that end, we complexed $4 \mu \mathrm{g}$ of mRNA with $4 \mu \mathrm{l}$ of a
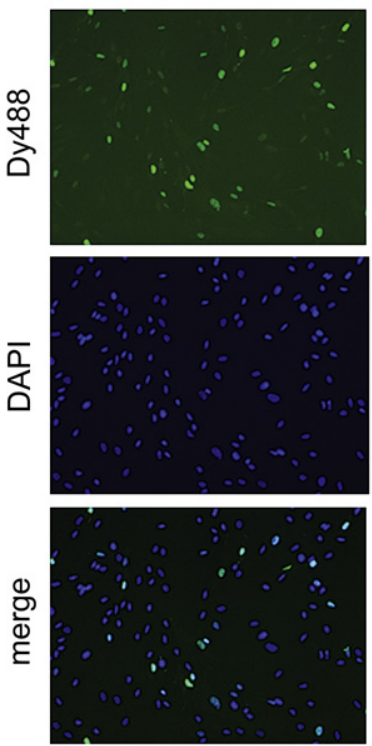

b
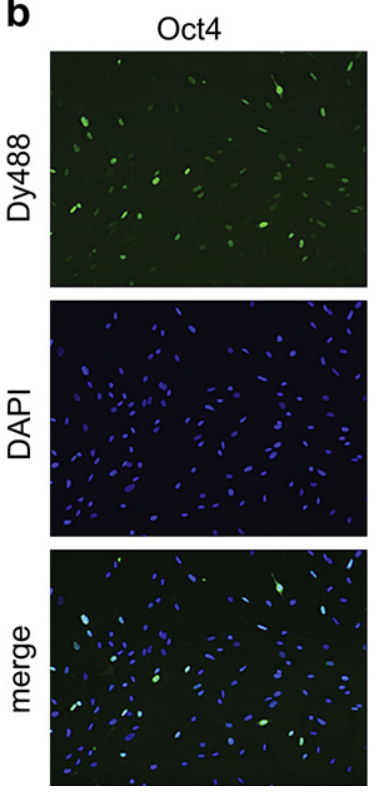

Sox 2
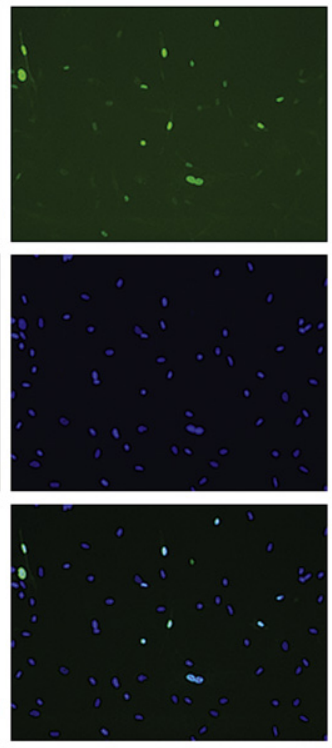

Sox2
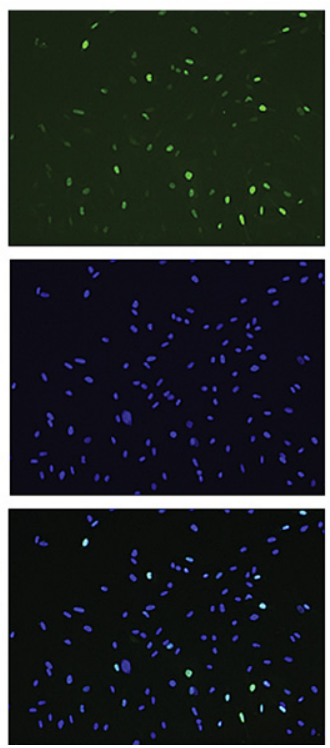

KIf4
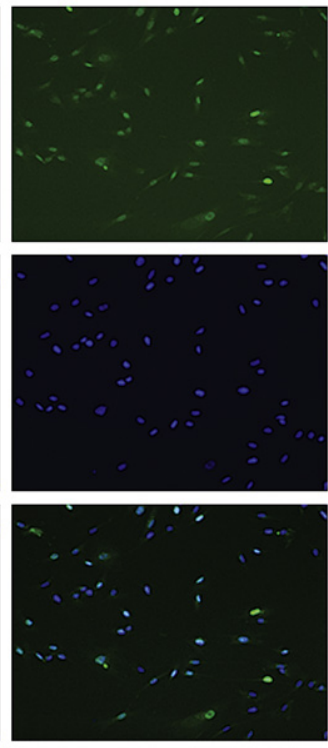

KIf4
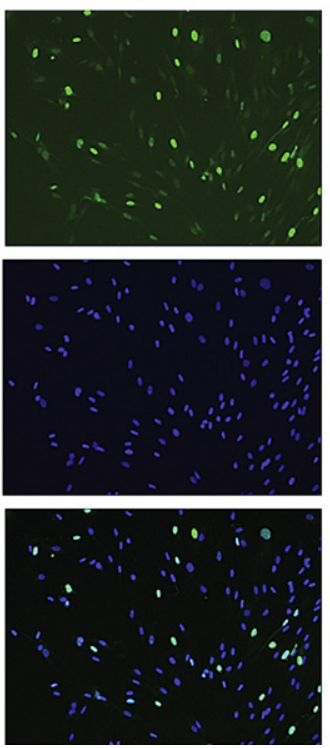

cMyc
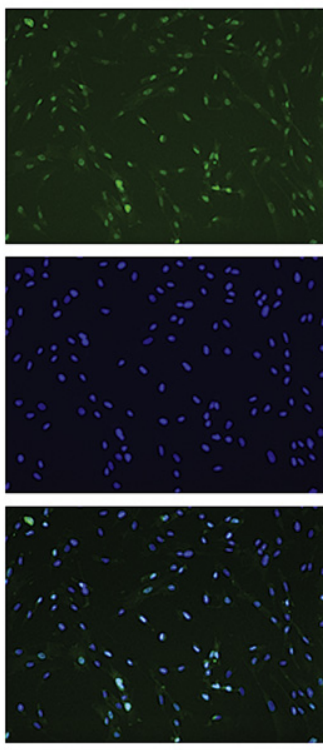

cMyc
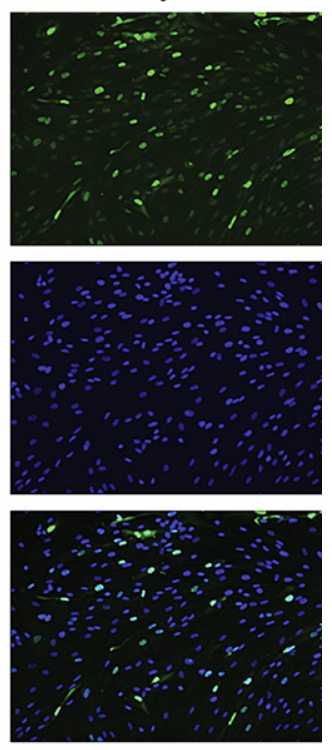

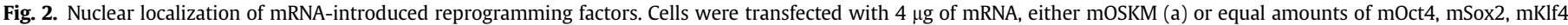

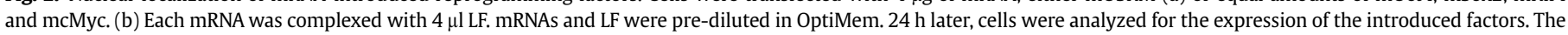
nuclei are stained with DAPI (magnification 40x). 


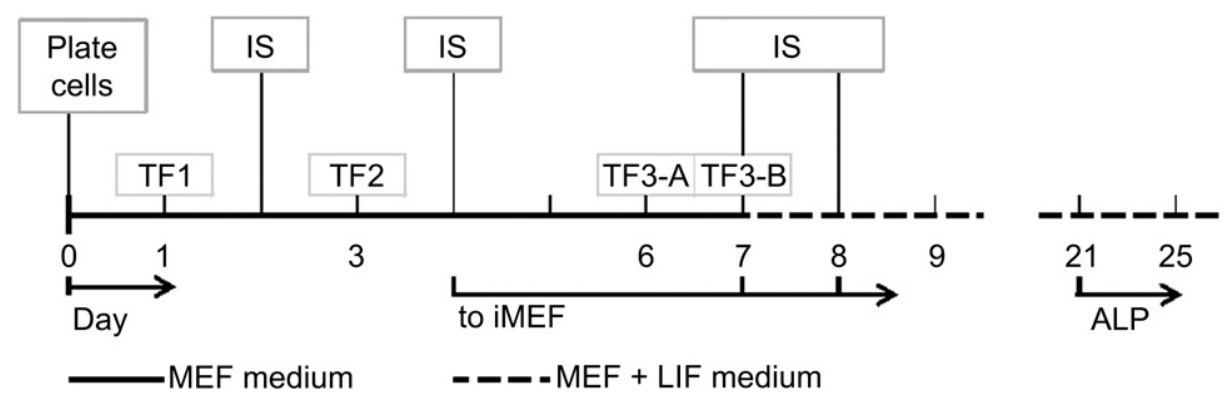

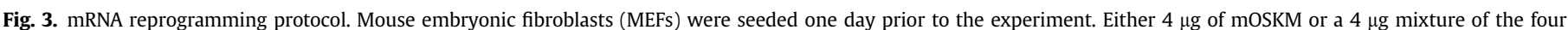

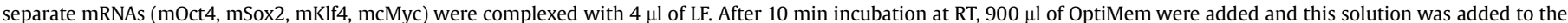

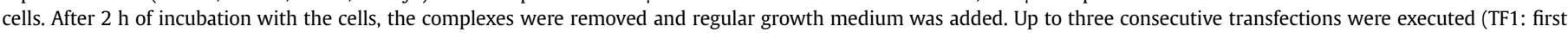

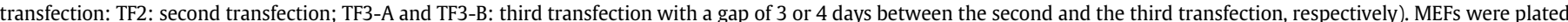

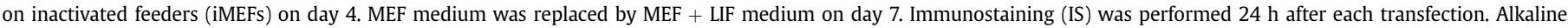
Phosphatase (ALP) staining was performed 19 days after each transfection.

Lipofectamine RNAiMAX (LF) as described above. One day after transfection, cells were fixed and stained for the four introduced factors. Microscopy analysis confirmed the correct intracellular localization of the reprogramming factors in the nucleus (Fig. 2).

\subsection{Quantitative analysis of the expression of reprogramming factors and pluripotency markers}

We transfected MEFs with $4 \mu \mathrm{g}$ mRNA, either mOSKM or equal amounts of mOct4, mSox2, mKlf4 and mcMyc, after complexation with $4 \mu \mathrm{l}$ of LF. We showed previously that our transfection method ensures sufficiently prolonged marker protein expression (up to eight days post transfection) [16]. However, to keep levels of reprogramming factors sufficiently high to induce reprogramming, we chose to perform multiple transfections. Keeping in mind the toxicity issues related to lipofectamine itself, we sought for a compromise between transfection frequency and acceptable cell survival. mRNA was introduced at day one (TF1), day three (TF2) and again at day six (TF3-A) or seven (TF3-B). On day four MEFs were plated onto inactivated feeders and on day seven MEF medium was replaced with MEF + LIF medium. Fig. 3 schematically shows when cells were plated on feeders and when immunostaining was performed. Twenty-four hours after each transfection, cells were analyzed by flow cytometry that followed intracellular immunostaining.

As shown in Fig. 4 significant levels of the reprogramming factors as well as two pluripotency markers (Nanog and SSEA-1) were detectable already after the first transfection and reached maximal levels after the third transfection performed at day 6 .

To further demonstrate the switch towards pluripotency, the cells were stained for alkaline phosphatase (ALP) 19 days after the last transfection. As shown in Fig. 5, ALP-positive cell clusters were clearly seen in wells, which were transfected twice (TF2) as well as in those transfected three times (TF3-A and TF3-B). This was not seen when cells were transfected only once.

\section{Discussion}

Successful transfection of MEFs with mGFP complexed with LF, with the majority of treated cells producing the marker protein, confirmed the efficiency of our transfection method. The method also proved to be effective in introducing mRNAs encoding Oct4, Sox2, Klf4, and cMyc into these cells. The expressed reprogramming factors were localized in the nucleus, which is a key requirement
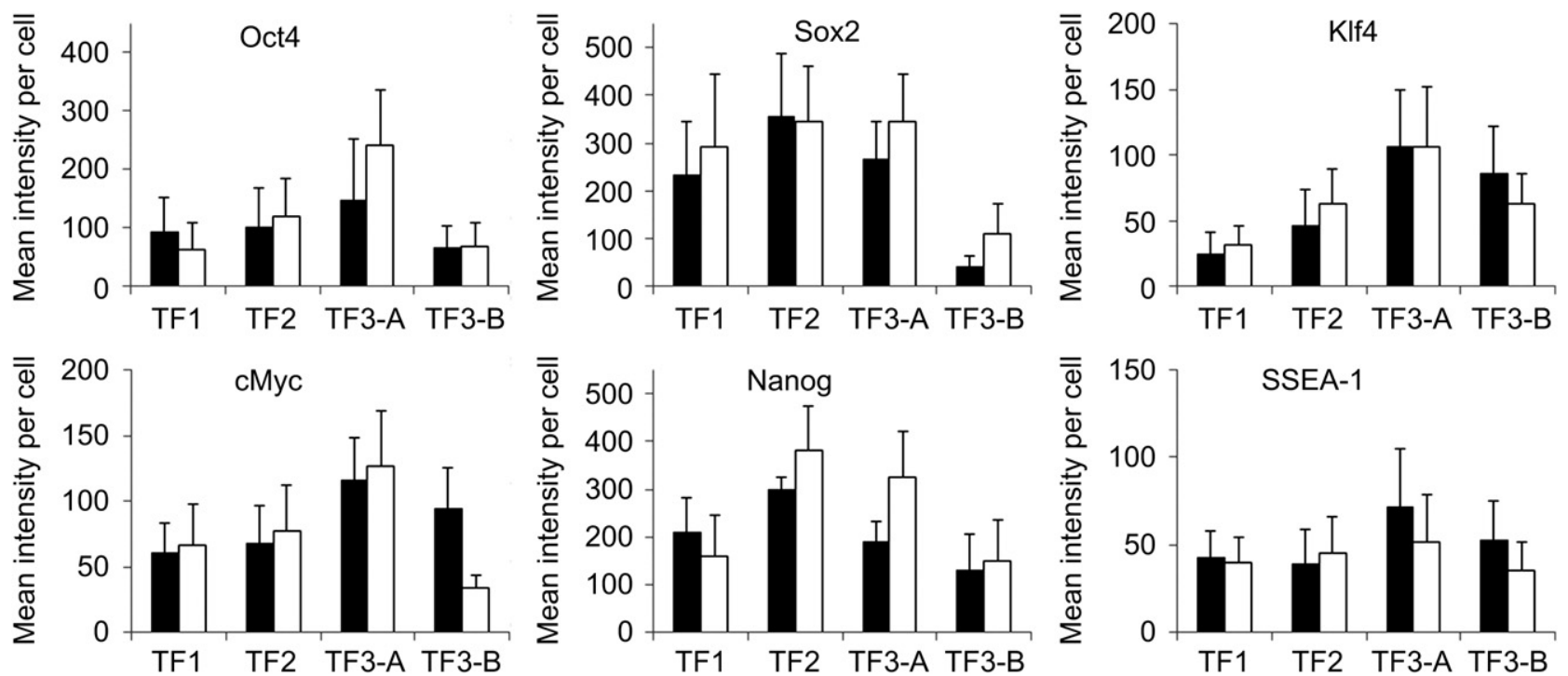

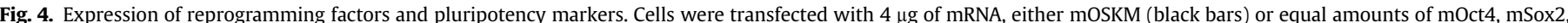

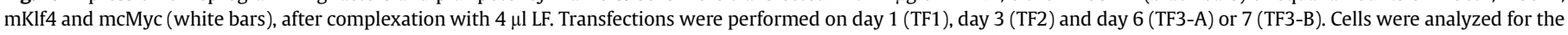
expression of the introduced factors and for Nanog and SSEA-1 24 h after each transfection. Bars represent means \pm SD, $n \geq 3$. 
TF2

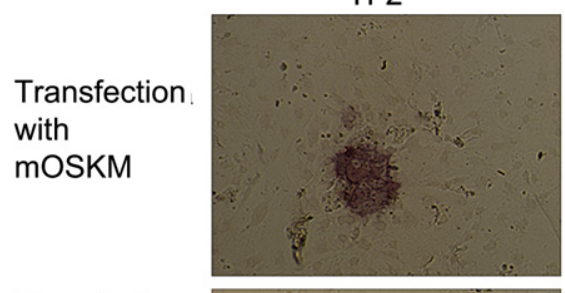

Transfection

with equal

amounts of

mOct4,

mSox2,

mKIf4 and

mcMyc
TF3-A
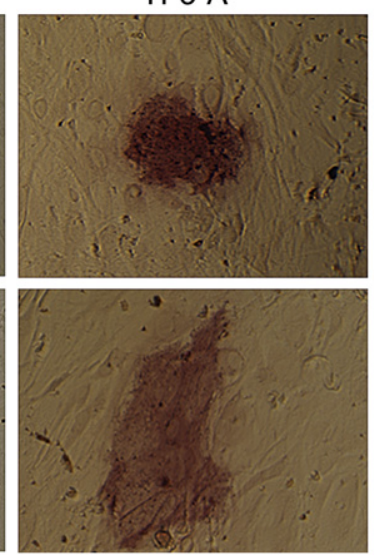

TF3-B
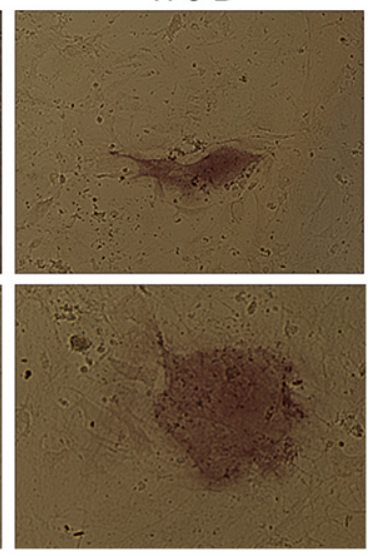

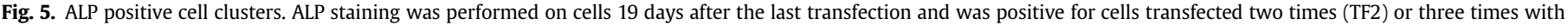
three days (TF3-A) or four days (TF3-B) between the second and third transfection (magnification 40x). Cells transfected only once (TF1) were ALP-negative.

for them to regulate the gene expression pattern towards pluripotency. Flow cytometry analysis confirmed the up-regulation of Oct4, Sox2, Klf4, cMyc, as well as Nanog and SSEA-1 in MEFs that were subjected to transfection with mRNAs encoding the Yamanaka factors (as a one mRNA-molecule or as equal amounts of the four mRNAs). Nanog and SSEA-1 are typically seen when MEFs transform into iPSCs [17] and this was already the case after one transfection. Interestingly, with a gap of four days (TF3-B) instead of three (TF3-A) between the second and third transfection, the levels of the introduced factors as well as Nanog and SSEA-1 were significantly lower, indicating that the level of the proteins should be maintained above a certain threshold for the induction of pluripotency. These results are in agreement with the findings of Jin et al. [18].

Moreover, the ALP activity was observed in cell clusters, which were transfected two or three times. This is a clear indication that the gene regulatory pattern inducing the reprogramming process in MEFs was switched on. This was not seen when cells were transfected only once, demonstrating once again that multiple transfections are required to maintain sufficient levels of the factors required to induce pluripotency in MEFs.

Our results are similar to those obtained by Plews and colleagues [13] who introduced mRNAs encoding reprogramming factors in human fibroblasts. The cells were transfected only once and electroporation was applied to deliver the mRNAs into the cells. The authors observed the up-regulation of both the transfected factors as well as the typical human pluripotency genes in the cells. This was demonstrated three days after electroporation and on day seven, when they observed even further increase in levels of endogenous OCT4, although levels of pluripotency marker genes were lower as compared to day three. This long term pattern of protein expression was somewhat unexpected since electroporation, due to which mRNA is delivered directly to the cytosol, is known to result in rather short protein expression [16].

Since it is clear that the transcription factors need to be expressed over a longer period of time, as we previously showed [16], a cationic lipid-mediated transfection method is preferable for this application. However, due to the toxicity mainly attributed to LF, lowering the amount of transfections needed to induce pluripotency, is an important issue to be addressed. To generate iPSCs, Warren et al. [14] lipofected human fibroblasts every day over a period of seventeen days. Such harsh transfection conditions induced substantial cell death. We could show that our transfection protocol allows less frequent transfections and still ensuring sufficient expression levels of the transcription factors as well as the pluripotency markers Nanog and SSEA-1. We both used LF to complex mRNAs implementing a similar protocol. The key difference however is the absence of serum during incubation with the cells in our method. We could demonstrate a substantial drop in transfection efficiency when serum is present during uptake of complexes ( $G$ Tavernier et al., manuscript submitted).

In our study we either used one mRNA-molecule encoding all four reprogramming factors separated by the viral peptide $2 \mathrm{~A}$ (mOSKM) or four different mRNAs each encoding one factor (mOct4, mSox2, mKlf4 and mcMyc). The mOSKM is constructed in a way that the four factors are translated in the transfected cells in a ratio that has been shown to be most efficient in generating iPSCs $[9,10]$. Our results do not show significant difference in the expression levels of the factors and pluripotency markers for both constructs. Nonetheless, cells transfected with the one-molecule mRNA did show a higher tolerance towards multiple transfections, looking healthier and showing higher proliferation capacity. This is likely due to the fact that more LF enters the cells when four separate mRNAs are delivered as compared to one larger mRNA strand.

\section{Conclusion}

Our data show that transfection of mouse embryonic fibroblasts with mRNAs encoding Yamanaka's factors can switch their fate towards pluripotency. All introduced factors were expressed at high levels and induced the expression of Nanog and SSEA-1, which are also markers that define pluripotency. Most importantly, our transfection method required merely two to three transfections to activate these pluripotency-associated genes. Positive ALP staining of the transfected cells further confirmed the onset of the reprogramming process. The mRNA-based approach, which avoids insertional mutagenesis, represents a promising method towards safe generation of iPSCs, which presents broad applicability for basic research, disease modelling and drug screening strategies.

\section{Acknowledgements}

Geertrui Tavernier is a doctoral fellow of the Institute for the Promotion of Innovation through Science and Technology in Flanders (IWT-Vlaanderen). We would also like to acknowledge this organization for founding the BRAINSTIM project. Katharina Wolfrum and James Adjaye acknowledge support from the Max Planck Society. The authors thank the scientific input of Prof. Dr. Catherine Verfaillie (Stem Cell Institute, KU Leuven). 


\section{References}

[1] Takahashi K, Yamanaka S. Induction of pluripotent stem cells from mouse embryonic and adult fibroblast cultures by defined factors. Cell 2006;126:663-76.

[2] Takahashi K, Tanabe K, Ohnuki M, Narita M, Ichisaka T, Tomoda K, et al. Induction of pluripotent stem cells from adult human fibroblasts by defined factors. Cell 2007;131:861-72.

[3] Yu J, Vodyanik MA, Smuga-Otto K, Antosiewicz-Bourget J, Frane JL, Tian S, et al. Induced pluripotent stem cell lines derived from human somatic cells. Science 2007;318:1917-20.

[4] Nakagawa M, Koyanagi M, Tanabe K, Takahashi K, Ichisaka T, Aoi T, et al. Generation of induced pluripotent stem cells without myc from mouse and human fibroblasts. Nat Biotechnol 2008;26:101-6.

[5] Huangfu D, Osafune K, Maehr R, Guo W, Eijkelenboom A, Chen S, et al. Induction of pluripotent stem cells from primary human fibroblasts with only Oct4 and Sox2. Nat Biotechnol 2008;126:1269-75.

[6] Li M, Sancho-Martinez I, Belmonte JCI. Cell fate conversion by mRNA. Stem Cell Res Ther 2011;2:5

[7] Stadtfeld M, Nagaya M, Utikal J, Weir G, Hochedlinger K. Induced pluripotent stem cells generated without viral integration. Science 2008;322:945-9.

[8] Yu J, Hu K, Smuga-Otto K, Tian S, Stewart R, Slukvin II , et al. Human induced pluripotent stem cells free of vector and transgene sequences. Science 2009; 324:797-801.

[9] Okita K, Nakagawa M, Hyenjong T, Yamanaka S. Generation of mouse induced pluripotent stem cells without viral vectors. Science 2008;322:949-53.

[10] Kaji K, Norrby K, Paca A, Mileikovsky M, Mohseni P, Woltjen K. Virus-free induction of pluripotency and subsequent excision of reprogramming factors. Nature $2009 \cdot 458 \cdot 771-5$.
[11] Sommer CA, Sommer AG, Longmire TA, Christodoulou C, Thomas DD, Gostissa $\mathrm{M}$, et al. Excision of reprogramming transgenes improves the differentiation potential of iPS cells generated with a single excisable vector. Stem Cells 2010;28:64-74.

[12] Zhou H, Wu S, Joo JY, Zhu S, Han DW, Lin T, et al. Generation of induced pluripotent stem cells using recombinant proteins. Cell Stem Cell 2009;4: 381-4.

[13] Plews JR, Li JL, Jones M, Moore HD, Mason C, Andrews P, et al. Activation of pluripotency genes in human fibroblast cells by a novel mRNA based approach. PLoS One 2010; 5.

[14] Warren L, Manos PD, Ahfeldt T, Loh YH, Li H, Lau F, et al. Highly efficient reprogramming to pluripotency and directed differentiation of human cells with synthetic modified mRNA. Cell Stem Cell 2010;7: 618-30.

[15] Nair SK, Boczkowski D, Morse M, Cumminig RI, Lyerly HK, Gilboa E. Induction of primary carcinoembryotic antigen (CEA)-specific cytotoxic T lymphocytes in vitro using human dendritic cells transfected with RNA. Nat Biotechnol 2008;16:364-9.

[16] Rejman J, Tavernier G, Bevarsad N, Demeester J, De Smedt SC. mRNA transfection of cervical carcinoma and mesenchymal stem cells mediated by cationic carriers. Journal of Control Release 2010;147:385-91.

[17] Mitsui K, Tokuzawa Y, Itoh H, Segawa K, Murakami M, Takahashi K, et al. The homeoprotein nanog is required for maintenance of pluripotency in mouse epiblast and ES cells. Cell 2003;113:631-42.

[18] Jin J, Kwon YW, Paek JS, Cho HJ, Lee JY, Chu IS, et al. Analysis of differential proteomes of induced pluripotent stem cells by protein-based reprogramming of fibroblasts. Journal of Proteome Research 2011;10: 977-89. 